J. Korean Math. Soc. 47 (2010), No. 1, pp. 1-15

DOI 10.4134/JKMS.2010.47.1.001

\title{
ON QUASI-STABLE EXCHANGE IDEALS
}

\author{
HuANYin Chen
}

\begin{abstract}
We introduce, in this article, the quasi-stable exchange ideal for associative rings. If $I$ is a quasi-stable exchange ideal of a ring $R$, then so is $M_{n}(I)$ as an ideal of $M_{n}(R)$. As an application, we prove that every square regular matrix over quasi-stable exchange ideal admits a diagonal reduction by quasi invertible matrices. Examples of such ideals are given as well.
\end{abstract}

\section{Introduction}

Following Ara (cf. [1]), an ideal $I$ of a ring $R$ is an exchange ideal provided that for every $x \in I$ there exist an idempotent $e \in I$ and elements $r, s \in I$ such that $e=x r=x+s-x s$. Clearly, an ideal $I$ of a $\operatorname{ring} R$ is an exchange ideal if and only if for any $x \in I$, there exists an idempotent $e \in x R$ such that $1-e \in(1-x) R$. Exchange ideal plays a key role in the direct sum decomposition theory of exchange rings. Many authors have studied such ideals, e.g., [1] and [12].

So as to investigate directly infinite rings, we introduce a new class of exchange ideals, i.e., quasi-stable exchange ideals of a ring $R$. If $I$ is a quasi-stable exchange ideal of a ring $R$, we will show that $M_{n}(I)$ is a quasi-stable exchange ideal of $M_{n}(R)$. As is well known, every square matrix over a unit-regular ring admits a diagonal reduction. Ara et. al. extended this result and proved that every square regular matrix over a separative exchange ring admits a diagonal reduction by invertible matrices (cf. [2]). It is interesting to investigate diagonal reduction of matrices over an ideal of a ring $R$ even though there exist some square matrices over $R$ which can not be reduced. As an application, we prove that every square regular matrix over quasi-stable exchange ideal admits a diagonal reduction by quasi invertible matrices. These also give nontrivial generalizations of [4, Theorem 16] and [6, Theorem 11].

Throughout, all rings are associative with identity, all ideals are two-sided ideals and all modules are right unitary modules. We use $M_{n}(R)$ to denote

Received February 15, 2007.

2000 Mathematics Subject Classification. 16E50, $19 \mathrm{~B} 10$.

Key words and phrases. quasi-stable ideal, exchange ideal, diagonal reduction. 
the ring of $n \times n$ matrices over $R$ with identity $I_{n}$. $G L_{n}(R)$ denotes the $n$ dimensional general linear group of $R$. Set $G L_{n}(I)=G L_{n}(R) \cap\left(I_{n}+M_{n}(I)\right)$. An element $x \in R$ is regular provided that $x=x y x$ for a $y \in R$. $\Gamma(I)$ stands for the set of all products of a left invertible element and a right invertible element in $1+I$, i.e., $\{u v \in R \mid \exists s, t \in 1+I$ such that $s u=1, v t=1\}$.

\section{Equivalent characterizations}

Definition 2.1. Let $I$ be an ideal of a $\operatorname{ring} R$. We say that $I$ is a right quasi-stable ideal if $a R+b R=R$ with $a \in I, b \in R$ implies that there exists $y \in R$ such that $a+b y \in \Gamma(I)$. We say that $I$ is a left quasi-stable ideal if $R a+R b=R$ with $a \in I, b \in R$ implies that there exists $z \in R$ such that $a+z b \in \Gamma(I)$. An ideal $I$ of a ring $R$ is a quasi-stable ideal in case it is both right and left quasi-stable ideal.

Let $J(R)$ be the Jacobson radical of rings $R$. If $a x+b=1$ with $a \in$ $J(R), x, b \in R$, then $b \in U(R)$. Hence, $a+b \cdot b^{-1}=1+J(R) \in \Gamma(J(R))$. Thus, $J(R)$ is a right quasi-stable exchange ideal. The purpose of this section is to investigate several equivalent characterizations of right quasi-stable ideals. The left quasi-stable ideals have analogous results.

Theorem 2.2. Let $I$ be an exchange ideal of a ring $R$. Then the following are equivalent:

(1) I is a right quasi-stable ideal.

(2) Every element in $I$ is a product of an idempotent in I and an element in $\Gamma(I)$.

Proof. $(1) \Rightarrow(2)$ Given any $x \in I$, there exists $y \in I$ such that $x=x y x$. Since $x y+(1-x y)=1$ with $x \in I$, we have $z \in R$ such that $x+(1-x y) z=w \in \Gamma(I)$. So $x=x y x=x y(x+(1-x y) z)=e w$, where $e=x y \in I$ is an idempotent.

$(2) \Rightarrow(1)$ Suppose that $a x+b=1$ with $a \in I, x, b \in R$. Then $b \in 1+I$. Since $I$ is an exchange ideal of $R$, by [1, Lemma 1.1], we have an idempotent $e=b s$ and $1-e=(1-b) t$ for some $s, t \in R$. Hence $a x t+e=1$, and then $(1-e) a x t+e=1$. So $(1-e) a \in I$ is regular. Thus we have an idempotent $f \in I$ and a $w \in \Gamma(I)$ such that $(1-e) a=f w$. So $f w x t+e=1$, and then $f w x t(1-f)+e(1-f)=1-f$. We infer that $f+e(1-f)=1-f w x t(1-f)$. Hence, $(1-e) a+e(1-f) w=f w+e(1-f) w=(1-f w x t(1-f)) w$. As a result, $a+b s((1-f) w-a)=(1+f w x t(1-f))^{-1} w \in \Gamma(I)$. Therefore $I$ is a right quasi-stable ideal.

Corollary 2.3. Let $I$ be an exchange ideal of a ring $R$. Then the following are equivalent:

(1) I is a right quasi-stable ideal.

(2) Whenever $a x+b=1$ with $a, x \in I, b \in 1+I$, there exists $y \in R$ such that $a+b y \in \Gamma(I)$. 
Proof. (1) $\Rightarrow(2)$ is trivial.

$(2) \Rightarrow(1)$ Let $x \in I$ be regular. Then we have $y \in I$ such that $x=x y x$. Since $x y+(1-x y)=1$ with $x, y \in I, 1-x y \in 1+I$, by hypothesis, there exists $z \in R$ such that $x+(1-x y) z=w \in \Gamma(I)$. Thus, $x=x y x=x y(x+(1-x y) z)=e w$, where $e=x y \in I$ is an idempotent. According to Theorem 2.2, we obtain the result.

Recall that an ideal $I$ of a ring $R$ has stable range one provided that $a R+$ $b R=R$ with $a \in I, b \in R$ implies that there exists $y \in R$ such that $a+b y \in$ $G L_{1}(R)$. We recall a simple known result.

Lemma 2.4. Given $a x+b=1, a, x, b \in R$, then the following hold:

(1) If $u(a+b y)=1$, then $(x+(1-x y) u b)(a+y(1-x a))=1$. If $(a+b y) u=1$, then $(a+y(1-x a))(x+(1-x y) u b)=1$.

(2) If $(x+z b) v=1$, then $(x+(1-x a) z)(a+b v(1-z a))=1$. If $v(x+z b)=1$, then $(a+b v(1-z a))(x+(1-x a) z)=1$.

Proof. Straightforward.

Proposition 2.5. Let $I$ be an exchange ideal of a ring $R$. If $I$ has stable range one, then $I$ is a right quasi-stable ideal

Proof. Assume that $a x+b=1$ with $a, x \in I, b \in 1+I$. Then $(a+(1-a) b)(x+$ $b)+(1-a) b(1-(x+b))=1$, where $a+(1-a) b \in 1+I$. Since $I$ has stable range one, we have $y \in R$ such that $(a+(1-a) b)+(1-a) b(1-(x+b)) y \in G L_{1}(I)$. That is, $a+(1-a) b(1+(1-(x+b)) y) \in G L_{1}(I)$. As $a(x+b)+(1-a) b=1$, we can find $z \in R$ such that $x+b+z(1-a) b \in G L_{1}(I)$, i.e., $x+(1+z(1-a)) b \in G L_{1}(I)$. By using Lemma 2.4 again, we have $t \in R$ such that $a+b t \in G L_{1}(I)$. Therefore $I$ is a right quasi-stable ideal, as desired.

It follows from Lemma 2.4 that stable range one for ideals is right and left symmetric. Recall that a ring $R$ is perfect in case $R / J(R)$ is a division ring and idempotents lift modulo $J(R)$. Consequently, every ideal of a perfect ring is quasi-stable.

Proposition 2.6. Let $I$ be an exchange ideal of a ring $R$. Then the following are equivalent:

(1) I is a right quasi-stable ideal.

(2) For any regular $a, b \in I, a R=b R$ implies that there exists $w \in \Gamma(I)$ such that $a=b w$.

Proof. (1) $\Rightarrow(2)$ Suppose that $a R=b R$ with regular $a, b \in I$. Then we have $x, y \in R$ such that $a x=b$ and $a=b y$. Assume that $b=b b^{\prime} b$. Replacing $b^{\prime} b y$ with $y$, we may assume that $y \in I$. From $y x+(1-y x)=1$, we have $z \in R$ such that $y+(1-y x) z=w \in \Gamma(I)$. Hence $a=b y=b(y+(1-y x) z)=b w$, as required. 
$(2) \Rightarrow(1)$ For any regular $x \in I$, there exists an idempotent $e \in I$ such that $x R=e R$. So $x=e w$ for some $w \in \Gamma(I)$. Therefore $I$ is a right quasi-stable ideal by Theorem 2.2 .

\section{Extensions of matrices}

A natural problem asks whether quasi-stable exchange ideal of a ring is invariant under matrix extension. In this section, we give this problem an affirmative answer. In the sequel, we say that the pair $(a, b)$ is an $I$-unimodular row in case $a x+b y=1$ for some $x \in I, y \in R$. The $I$-unimodular row $(a, b)$ is called $I$-reducible if there exists $z \in R$ such that $a+b z \in \Gamma(I)$.

Lemma 3.1. Let $(a, b)$ be a I-unimodular row in a ring $R$. Let $u, v \in G L_{1}(I)$ and $c \in R$. Then $(v a u+v b c, v b)$ is also $I$-unimodular row. Furthermore, $(a, b)$ is I-reducible if and only if so is $(v a u+v b c, v b)$.

Proof. Since $(a, b)$ is an $I$-unimodular row in a ring $R$, we have $x \in I, y \in R$ such that $a x+b y=1$. Hence $(v a u+v b c)\left(u^{-1} x v^{-1}\right)+v b\left(y-c u^{-1} x\right) v^{-1}=1$. Clearly, $u^{-1} x v^{-1} \in I$. So $(v a u+v b c, v b)$ is an $I$-unimodular row. Assume that $(a, b)$ is $I$-reducible. Then we have $y \in R$ such that $a+b y \in \Gamma(I)$. Choose $z=y u-c$. Then we see that $(v a u+v b c)+(v b) z=v(a+b y) u \in \Gamma(I)$; hence, $(a u+v b c, v b)$ is $I$-reducible. Conversely, assume that there exists $z \in R$ such that $v a u+v b c+v b z \in \Gamma(I)$. Then $v\left(a+b(c+z) u^{-1}\right) u \in \Gamma(I)$. As $u, v \in G L_{1}(I)$, $a+b(c+z) u^{-1} \in \Gamma(I)$. Therefore $(a, b)$ is $I$-reducible.

Theorem 3.2. Let $I$ be a right quasi-stable exchange ideal of a ring $R$. Then $M_{n}(I)$ is a right quasi-stable exchange ideal of $M_{n}(R)$ for all $n \in \mathbb{N}$.

Proof. By [1, Theorem 1.4], $M_{n}(I)$ is an exchange ideal of $M_{n}(R)$. We now induct on $n$. Assume inductively that the result holds for $n$. It will suffice to show that the result holds for $n+1$. Suppose that

(*)

$$
\begin{aligned}
&\left(\begin{array}{cccc}
a_{11} & a_{12} & \cdots & a_{1(n+1)} \\
a_{21} & a_{22} & \cdots & a_{2(n+1)} \\
\vdots & \vdots & \ddots & \vdots \\
a_{(n+1) 1} & a_{(n+1) 2} & \cdots & a_{(n+1)(n+1)}
\end{array}\right)\left(\begin{array}{cccc}
b_{11} & b_{12} & \cdots & b_{1(n+1)} \\
b_{21} & b_{22} & \cdots & b_{2(n+1)} \\
\vdots & \vdots & \ddots & \vdots \\
b_{(n+1) 1} & b_{(n+1) 2} & \cdots & b_{(n+1)(n+1)}
\end{array}\right) \\
&+\left(\begin{array}{cccc}
c_{11} & c_{12} & \cdots & c_{1(n+1)} \\
c_{21} & c_{22} & \cdots & c_{2(n+1)} \\
\vdots & \vdots & \ddots & \vdots \\
c_{(n+1) 1} & c_{(n+1) 2} & \cdots & c_{(n+1)(n+1)}
\end{array}\right)=I_{n+1}
\end{aligned}
$$

in $M_{n+1}(R)$, where

$$
\left(\begin{array}{ccc}
a_{11} & \cdots & a_{1(n+1)} \\
a_{21} & \cdots & a_{2(n+1)} \\
\vdots & \ddots & \vdots \\
a_{(n+1) 1} & \cdots & a_{(n+1)(n+1)}
\end{array}\right),\left(\begin{array}{ccc}
b_{11} & \cdots & b_{1(n+1)} \\
b_{21} & \cdots & b_{2(n+1)} \\
\vdots & \ddots & \vdots \\
b_{(n+1) 1} & \cdots & b_{(n+1)(n+1)}
\end{array}\right) \in M_{n+1}(I) .
$$


Then $a_{11} b_{11}+a_{12} b_{21}+\cdots+a_{1(n+1)} b_{(n+1) 1}+c_{11}=1$ with $a_{11} \in I$. As $I$ is a quasi-stable exchange ideal of $R$, we have $z_{1} \in R$ such that $a_{11}+\left(a_{12} b_{21}+\cdots+\right.$ $\left.a_{1 n} b_{n 1}+c_{11}\right) z_{1} \in \Gamma(I)$. Since

$$
\left(\begin{array}{ccccc}
1 & 0 & 0 & \cdots & 0 \\
b_{21} z_{1} & 1 & 0 & \cdots & 0 \\
b_{31} z_{1} & 0 & 1 & \cdots & 0 \\
\vdots & \vdots & \vdots & \ddots & \vdots \\
b_{(n+1) 1} z_{1} & 0 & 0 & \cdots & 1
\end{array}\right) \in G L_{n+1}(I)
$$

by virtue of Lemma 3.1, (*) is $M_{n+1}(I)$-reducible if and only if this is so for the $M_{n+1}(I)$-unimodular row with elements

$$
\begin{gathered}
\left(\begin{array}{ccccc}
a_{11} & a_{12} & a_{13} & \cdots & a_{1(n+1)} \\
a_{21} & a_{22} & a_{23} & \cdots & a_{2(n+1)} \\
a_{31} & a_{32} & a_{33} & \cdots & a_{3(n+1)} \\
\vdots & \vdots & \vdots & \ddots & \vdots \\
a_{(n+1) 1} & a_{(n+1) 2} & a_{(n+1) 3} & \cdots & a_{(n+1)(n+1)}
\end{array}\right)\left(\begin{array}{ccccc}
1 & 0 & 0 & \cdots & 0 \\
b_{21} z_{1} & 1 & 0 & \cdots & 0 \\
b_{31} z_{1} & 0 & 1 & \cdots & 0 \\
\vdots & \vdots & \vdots & \ddots & \vdots \\
b_{(n+1) 1} z_{1} & 0 & 0 & \cdots & 1
\end{array}\right) \\
+\left(\begin{array}{ccccc}
c_{11} & c_{12} & c_{13} & \cdots & c_{1(n+1)} \\
c_{21} & c_{22} & c_{23} & \cdots & c_{2(n+1)} \\
c_{31} & c_{32} & c_{33} & \cdots & c_{3(n+1)} \\
\vdots & \vdots & \vdots & \ddots & \vdots \\
c_{(n+1) 1} & c_{(n+1) 2} & c_{(n+1) 3} & \cdots & c_{(n+1)(n+1)}
\end{array}\right)\left(\begin{array}{ccccc}
z_{1} & 0 & 0 & \cdots & 0 \\
0 & 0 & 0 & \cdots & 0 \\
0 & 0 & 0 & \cdots & 0 \\
\vdots & \vdots & \vdots & \ddots & \vdots \\
0 & 0 & 0 & \cdots & 0
\end{array}\right)
\end{gathered}
$$

and

$$
\left(\begin{array}{ccccc}
c_{11} & c_{12} & c_{13} & \cdots & c_{1(n+1)} \\
c_{21} & c_{22} & c_{23} & \cdots & c_{2(n+1)} \\
c_{31} & c_{32} & c_{33} & \cdots & c_{3(n+1)} \\
\vdots & \vdots & \vdots & \ddots & \vdots \\
c_{(n+1) 1} & c_{(n+1) 2} & c_{(n+1) 3} & \cdots & c_{(n+1)(n+1)}
\end{array}\right)
$$

So we assume that $a_{11} \in \Gamma(I)$. From $c_{21}, \ldots, c_{(n+1) 1} \in I$, we have $a_{i j} \in I$ (either $i \neq 1$ or $j \neq 1$ ) in $(*)$. Write $a_{11}=u v, s u=1$, vt $=1, s, t \in 1+I$. Then $s a_{11} t=1$, and so

$$
\left(\begin{array}{ccccc}
s & 0 & 0 & \cdots & 0 \\
1-a_{11} t s & a_{11} t & 0 & \cdots & 0 \\
0 & 0 & 1 & \cdots & 0 \\
\vdots & \vdots & \vdots & \ddots & \vdots \\
0 & 0 & 0 & \cdots & 1
\end{array}\right)
$$




$$
\begin{aligned}
& \times\left(\begin{array}{ccccc}
a_{11} & a_{12} & a_{13} & \cdots & a_{1(n+1)} \\
a_{21} & a_{22} & a_{23} & \cdots & a_{2(n+1)} \\
a_{31} & a_{32} & a_{33} & \cdots & a_{3(n+1)} \\
\vdots & \vdots & \vdots & \ddots & \vdots \\
a_{(n+1) 1} & a_{(n+1) 2} & a_{(n+1) 3} & \cdots & a_{(n+1)(n+1)}
\end{array}\right) \\
& \times\left(\begin{array}{ccccc}
t & 1-t s a_{11} & 0 & \cdots & 0 \\
0 & s a_{11} & 0 & \cdots & 0 \\
0 & 0 & 1 & \cdots & 0 \\
\vdots & \vdots & \vdots & \ddots & \vdots \\
0 & 0 & 0 & \cdots & 1
\end{array}\right) \\
& =\left(\begin{array}{ccccc}
1 & d_{12} & d_{13} & \cdots & d_{1(n+1)} \\
d_{21} & d_{22} & d_{23} & \cdots & d_{2(n+1)} \\
d_{31} & d_{32} & d_{33} & \cdots & d_{3(n+1)} \\
\vdots & \vdots & \vdots & \ddots & \vdots \\
d_{(n+1) 1} & d_{(n+1) 2} & d_{(n+1) 3} & \cdots & d_{(n+1)(n+1)}
\end{array}\right),
\end{aligned}
$$

where

$$
\begin{aligned}
& \left(\begin{array}{ccccc}
s & 0 & 0 & \cdots & 0 \\
1-a_{11} t s & a_{11} t & 0 & \cdots & 0 \\
0 & 0 & 1 & \cdots & 0 \\
\vdots & \vdots & \vdots & \ddots & \vdots \\
0 & 0 & 0 & \cdots & 1
\end{array}\right)=\left(\begin{array}{ccccc}
a_{11} t & 1-a_{11} t s & 0 & \cdots & 0 \\
0 & s & 0 & \cdots & 0 \\
0 & 0 & 1 & \cdots & 0 \\
\vdots & \vdots & \vdots & \ddots & \vdots \\
0 & 0 & 0 & \cdots & 1
\end{array}\right)^{-1} \\
& \left(\begin{array}{ccccc}
t & 1-t s a_{11} & 0 & \cdots & 0 \\
0 & s a_{11} & 0 & \cdots & 0 \\
0 & 0 & 1 & \cdots & 0 \\
\vdots & \vdots & \vdots & \ddots & \vdots \\
0 & 0 & 0 & \cdots & 1
\end{array}\right)=\left(\begin{array}{ccccc}
s a_{11} & 0 & 0 & \cdots & 0 \\
1-t s a_{11} & t & 0 & \cdots & 0 \\
0 & 0 & 1 & \cdots & 0 \\
\vdots & \vdots & \vdots & \ddots & \vdots \\
0 & 0 & 0 & \cdots & 1
\end{array}\right)^{-1} \in G L_{n+1}(I) .
\end{aligned}
$$

Thus (*) is $M_{n+1}(I)$-reducible if and only if this is so for the $M_{n+1}(I)$-unimodular row with elements

$$
\begin{aligned}
& \left(\begin{array}{ccccc}
1 & d_{12} & d_{13} & \cdots & d_{1(n+1)} \\
d_{21} & d_{22} & d_{23} & \cdots & d_{2(n+1)} \\
d_{31} & d_{32} & * & \cdots & * \\
\vdots & \vdots & \vdots & \ddots & \vdots \\
d_{(n+1) 1} & d_{3(n+1)} & * & \cdots & d_{(n+1)(n+1)}
\end{array}\right) \\
& \left(\begin{array}{ccccc}
s & 0 & 0 & \cdots & 0 \\
1-a_{11} t s & a_{11} t & 0 & \cdots & 0 \\
0 & 0 & 1 & \cdots & 0 \\
\vdots & \vdots & \vdots & \ddots & \vdots \\
0 & 0 & 0 & \cdots & 1
\end{array}\right)\left(\begin{array}{ccccc}
c_{11} & c_{12} & c_{13} & \cdots & c_{1(n+1)} \\
c_{21} & c_{22} & c_{23} & \cdots & c_{2(n+1)} \\
c_{31} & c_{32} & c_{33} & \cdots & c_{3(n+1)} \\
\vdots & \vdots & \vdots & \ddots & \vdots \\
c_{(n+1) 1} & c_{(n+1) 2} & c_{(n+1) 3} & \cdots & c_{(n+1)(n+1)}
\end{array}\right) .
\end{aligned}
$$


In (*), we may assume that $d_{i j} \in I$ (either $3 \leq i \leq n+1$ or $3 \leq j \leq n+1$ ) and $d_{12}=s a_{11}\left(1-t s a_{11}\right)+s a_{12} s a_{11}, d_{21}=\left(1-a_{11} t s\right) a_{11} t+a_{11} t a_{21} t, d_{22}=$ $\left(\left(1-a_{11} t s\right) a_{11}+a_{11} t a_{21}\right)\left(1-t s a_{11}\right)+\left(\left(1-a_{11} t s\right) a_{12}+a_{11} t a_{22}\right) s a_{11} \in I$. By Lemma 3.1 again, $(*)$ is $M_{n+1}(I)$-reducible if and only if this is so for the $M_{n+1}(I)$-unimodular row with elements

$$
\begin{aligned}
& \left(\begin{array}{ccccc}
1 & 0 & 0 & \cdots & 0 \\
0 & * & * & \cdots & * \\
0 & * & * & \cdots & * \\
\vdots & \vdots & \vdots & \ddots & \vdots \\
0 & * & * & \cdots & *
\end{array}\right), \\
& \left(\begin{array}{ccccc}
1 & 0 & 0 & \cdots & 0 \\
* & 1 & 0 & \cdots & 0 \\
* & 0 & 1 & \cdots & 0 \\
\vdots & \vdots & \vdots & \ddots & \vdots \\
* & 0 & 0 & \cdots & 1
\end{array}\right)\left(\begin{array}{ccccc}
s & 0 & 0 & \cdots & 0 \\
1-a_{11} t s & a_{11} t & 0 & \cdots & 0 \\
0 & 0 & 1 & \cdots & 0 \\
\vdots & \vdots & \vdots & \ddots & \vdots \\
0 & 0 & 0 & \cdots & 1
\end{array}\right)\left(\begin{array}{ccccc}
c_{11} & c_{12} & c_{13} & \cdots & c_{1(n+1)} \\
c_{21} & c_{22} & c_{23} & \cdots & c_{2(n+1)} \\
c_{31} & c_{32} & c_{33} & \cdots & c_{3(n+1)} \\
\vdots & \vdots & \vdots & \ddots & \vdots \\
c_{(n+1) 1} & c_{(n+1) 2} & c_{(n+1) 3} & \cdots & c_{(n+1)(n+1)}
\end{array}\right) .
\end{aligned}
$$

So we may assume that $a_{11}=1, a_{1 i}=0=a_{i 1}(2 \leq i \leq n+1)$ in $(*)$. Furthermore, we may assume that $(*)$ is in the following form:

$$
\left(\begin{array}{cc}
1 & 0_{1 \times n} \\
0_{n \times 1} & D
\end{array}\right)\left(\begin{array}{cc}
e_{11} & E_{12} \\
E_{21} & E_{22}
\end{array}\right)+\left(\begin{array}{cc}
c_{11} & C_{12} \\
C_{21} & C_{22}
\end{array}\right)=\left(\begin{array}{cc}
1 & 0 \\
0 & I_{n}
\end{array}\right),
$$

$D \in M_{n}(I)$ and $\left(\begin{array}{ll}e_{11} & E_{12} \\ E_{21} & E_{22}\end{array}\right) \in M_{n+1}(I)$. This infers that $D E_{22}+C_{22}=I_{n}$. By the induction hypothesis, $M_{n}(I)$ is a quasi-stable exchange ideal of $M_{n}(R)$. So we can find $Z_{2} \in M_{n}(R)$ such that $D+C_{22} Z_{2} \in \Gamma\left(M_{n}(I)\right)$. Thus, we pass to the $M_{n+1}(I)$-unimodular row with elements

$$
\left(\begin{array}{cc}
1 & 0_{1 \times n} \\
0_{n \times 1} & D
\end{array}\right)+\left(\begin{array}{ll}
c_{11} & C_{12} \\
C_{21} & C_{22}
\end{array}\right)\left(\begin{array}{cc}
0 & 0_{1 \times n} \\
0_{n \times 1} & Z_{2}
\end{array}\right),\left(\begin{array}{ll}
c_{11} & C_{12} \\
C_{21} & C_{22}
\end{array}\right) .
$$

In addition, we have $C_{12} \in M_{1 \times n}(I)$. It suffices to prove that $M_{n+1}(I)$ unimodular row with elements

$$
\left(\begin{array}{cc}
1 & C_{12} Z_{2} \\
0_{n \times 1} & D+C_{22} Z_{2}
\end{array}\right) \text { and }\left(\begin{array}{cc}
c_{11} & C_{12} \\
C_{21} & C_{22}
\end{array}\right)
$$

is $M_{n+1}(I)$-reducible. Write $D+C_{22} Z_{2}=U V, S U=I_{n}, V T=I_{n}, S, T$ $\in I_{n}+M_{n}(I)$. Thus,

$$
\begin{aligned}
& \left(\begin{array}{cc}
1 & C_{12} Z_{2} \\
0_{n \times 1} & D+C_{22} Z_{2}
\end{array}\right)=\left(\begin{array}{cc}
1 & 0_{1 \times n} \\
0_{n \times 1} & U
\end{array}\right)\left(\begin{array}{cc}
1 & C_{12} Z_{2} \\
0_{n \times 1} & V
\end{array}\right), \\
& \left(\begin{array}{cc}
1 & 0_{1 \times n} \\
0_{n \times 1} & S
\end{array}\right)\left(\begin{array}{cc}
1 & 0_{1 \times n} \\
0_{n \times 1} & U
\end{array}\right)=I_{n+1}, \\
& \left(\begin{array}{cc}
1 & C_{12} Z_{2} \\
0_{n \times 1} & V
\end{array}\right)\left(\begin{array}{cc}
1 & 0_{1 \times n} \\
0_{n \times 1} & T
\end{array}\right)\left(\begin{array}{cc}
1 & -C_{12} Z_{2} T \\
0_{n \times 1} & I_{2}
\end{array}\right)=I_{n+1}, \\
& \left(\begin{array}{cc}
1 & 0_{1 \times n} \\
0_{n \times 1} & S
\end{array}\right),\left(\begin{array}{cc}
1 & 0_{1 \times n} \\
0_{n \times 1} & T
\end{array}\right)\left(\begin{array}{cc}
1 & -C_{12} Z_{2} T \\
0_{n \times 1} & I_{2}
\end{array}\right) \in I_{n+1}+M_{n+1}(I) .
\end{aligned}
$$


This implies that $\left(\begin{array}{cc}1 & C_{12} Z_{2} \\ 0 & D+C_{22} Z_{2}\end{array}\right) \in \Gamma\left(M_{n+1}(I)\right)$, as required.

Corollary 3.3. Let $I$ be a right quasi-stable exchange ideal of a ring $R$. Then every regular $n \times n$ matrix over $I$ is a product of an idempotent $n \times n$ matrix over $I$ and an matrix in $\Gamma\left(M_{n}(I)\right)$.

Proof. Since $I$ is a right quasi-stable exchange ideal of $R$, by Theorem 3.2, $M_{n}(I)$ is a right quasi-stable exchange ideal of $M_{n}(R)$. Therefore we complete the proof from Theorem 2.2.

Let $F P(I)$ denote the set of finitely generated projective right $R$-module $P$ such that $P=P I$.

Lemma 3.4. Let $I$ be an exchange ideal of a ring $R$. If $P \in F P(I)$. Then there exist idempotents $e_{1}, \ldots, e_{n} \in I$ such that $P \cong e_{1} R \oplus \cdots \oplus e_{n} R$.

Proof. See [1, Proposition 1.5].

Lemma 3.5. Let $I$ be a quasi-stable exchange ideal of a ring $R$. For any regular $a, b \in I, a R \cong b R$ implies that $a=w_{1}$ bw $w_{2}$ for some $w_{1}, w_{2} \in \Gamma(I)$.

Proof. Suppose that $\psi: a R \cong b R$. Then one easily checks that $R a=R \psi(a)$ and $\psi(a) R=b R$. As $a \in I$, we have $\psi(a) \in R a \subseteq I$. Since $I$ is a right quasistable ideal, it follows by Proposition 2.6 that there exists $w_{2} \in \Gamma(I)$ such that $b w_{2}=\psi(a)$. Likewise, we have $w_{1} \in \Gamma(I)$ such that $a=w_{1} \psi(a)$. Therefore $a=w_{1} b w_{2}$, where $w_{1}, w_{2} \in \Gamma(I)$.

We use $A^{T}$ to denote the transpose of the matrix $A$. We now derive the main result of this article.

Theorem 3.6. Let I be a quasi-stable exchange ideal of a ring $R$. Then every square regular matrix over I admits a diagonal reduction by quasi invertible matrices.

Proof. Given any regular $A \in M_{n}(I)$, we have an idempotent matrix $E \in$ $M_{n}(I)$ such that $A R^{n \times 1}=E^{n \times 1} R^{n \times 1}$, where $R^{n \times 1}=\left\{\left(x_{1}, \ldots, x_{n}\right)^{T} \mid x_{1}, \ldots\right.$, $\left.x_{n} \in R\right\}$. Clearly, $E R^{n \times 1} \in F P(I)$. By Lemma 3.4, there exist idempotents $e_{1}, \ldots, e_{n} \in I$ such that $E R^{n \times 1} \cong e_{1} R \oplus \cdots \oplus e_{n} R \cong \operatorname{diag}\left(e_{1}, \ldots, e_{n}\right) R^{n \times 1}$ as right $R$-modules. Set $R^{1 \times n}=\left\{\left(x_{1}, \ldots, x_{n}\right) \mid x_{1}, \ldots, x_{n} \in R\right\}$. Then $A R^{n \times 1} \bigotimes_{R} R^{1 \times n} \cong \operatorname{diag}\left(e_{1}, \ldots, e_{n}\right) R^{n \times 1} \bigotimes_{R} R^{1 \times n}$. So $A M_{n}(R) \cong \operatorname{diag}\left(e_{1}, \ldots\right.$, $\left.e_{n}\right) M_{n}(R)$. Therefore the result follows.

Let $I$ be an ideal of a ring $R$. We use $T M_{n}(R)$ to denote the ring of all $n \times n$ lower triangular matrices over $R$ and $T M_{n}(I)$ to denote the ideal of all $n \times n$ lower triangular matrices over $I$.

Lemma 3.7. Let $I$ be an ideal of a ring $R$, and let $n \in \mathbb{N}$. If $u_{i i} \in \Gamma(I)(1 \leq$ $i \leq n), u_{i j} \in I(j<i, 1 \leq i, j \leq n)$ and $u_{i j}=0(i<j, 1 \leq i, j \leq n)$. Then $\left(u_{i j}\right)_{n \times n} \in \Gamma\left(T M_{n}(I)\right)$. 
Proof. Straightforward.

Proposition 3.8. Let $I$ be a right quasi-stable exchange ideal of a ring $R$, and let $n \in \mathbb{N}$. Then $T M_{n}(I)$ is a right quasi-stable exchange ideal of $T M_{n}(R)$.

Proof. Obviously, $T M_{n}(I)$ is an exchange ideal of $T M_{n}(R)$. Given

$$
\left(\begin{array}{ccc}
a_{1} & \cdots & 0 \\
\vdots & \ddots & \vdots \\
* & \cdots & a_{n}
\end{array}\right)\left(\begin{array}{ccc}
x_{1} & \cdots & 0 \\
\vdots & \ddots & \vdots \\
* & \cdots & x_{n}
\end{array}\right)+\left(\begin{array}{ccc}
b_{1} & \cdots & 0 \\
\vdots & \ddots & \vdots \\
* & \cdots & b_{n}
\end{array}\right)=I_{n}
$$

with $\left(\begin{array}{ccc}a_{1} & \cdots & 0 \\ \vdots & \ddots & \vdots \\ * & \cdots & a_{n}\end{array}\right),\left(\begin{array}{ccc}x_{1} & \cdots & 0 \\ \vdots & \ddots & \vdots \\ * & \cdots & x_{n}\end{array}\right) \in T M_{n}(I)$, then for each $i(1 \leq i \leq n)$ we get $a_{i i} x_{i i}+b_{i i}=1$ with $a_{i i} \in I, x_{i i}, b_{i i} \in R$. As $I$ is a right quasi-stable ideal, we can find $y_{i} \in R$ such that $a_{i i}+b_{i i} y_{i} \in \Gamma(I)$. Clearly, $b_{i i} \in 1+I$ and $a_{i j}, b_{i j} \in I(j<i, 1 \leq i, j \leq n)$. By virtue of Lemma 3.7, we get

$$
\begin{aligned}
& \left(\begin{array}{ccc}
a_{1} & \cdots & 0 \\
\vdots & \ddots & \vdots \\
* & \cdots & a_{n}
\end{array}\right)+\left(\begin{array}{ccc}
b_{1} & \cdots & 0 \\
\vdots & \ddots & \vdots \\
* & \cdots & b_{n}
\end{array}\right)\left(\begin{array}{ccc}
y_{1} & \cdots & 0 \\
\vdots & \ddots & \vdots \\
0 & \cdots & y_{n}
\end{array}\right) \\
= & \left(\begin{array}{ccc}
a_{11}+b_{11} y_{1} & \cdots & 0 \\
\vdots & \ddots & \vdots \\
* & \cdots & a_{n n}+b_{n n} y_{n}
\end{array}\right) \in \Gamma\left(T M_{n}(I)\right),
\end{aligned}
$$

as required.

\section{Examples}

The aim of this section is to construct several examples of quasi-stable ideals. A natural problem asks that if right quasi-stable ideal is right and left symmetric. So far, we can not answer this question. Now we establish an interesting properties of such ideals, which is an extension of [4, Lemma 14].

Proposition 4.1. Let $I$ be a right quasi-stable ideal of a ring $R$. Then for any regular $x \in I$, there exist an idempotent $e \in R$, a right invertible $u \in 1+I$, a left invertible $v \in 1+I$ such that $x=e u v$.

Proof. Assume that $A=\left(a_{i j}\right) \in G L_{2}(R) \cap\left(\begin{array}{cc}1+I & 1+I \\ I & 1+I\end{array}\right)$, where $a_{12} \in \Gamma(I)$. Write $a_{12}=u v, s u=1, v t=1, s, t \in 1+I$. Then $s a_{12} t=1$. Clearly, we have

$$
\begin{aligned}
\left(\begin{array}{cc}
s & 0 \\
1-a_{12} t s & a_{12} t
\end{array}\right) & =\left(\begin{array}{cc}
a_{12} t & 1-a_{12} t s \\
0 & s
\end{array}\right)^{-1}, \\
\left(\begin{array}{cc}
s a_{12} & 0 \\
1-t s a_{12} & t
\end{array}\right) & =\left(\begin{array}{cc}
t & 1-t s a_{12} \\
0 & s a_{12}
\end{array}\right)^{-1} \in G L_{2}(I) .
\end{aligned}
$$


So we get

$$
\begin{aligned}
& \quad\left(\begin{array}{cc}
s & 0 \\
1-a_{12} t s & a_{12} t
\end{array}\right) A\left(\begin{array}{cc}
s a_{12} & 0 \\
1-t s a_{12} & t
\end{array}\right)=\left(\begin{array}{cc}
* & 1 \\
* & *
\end{array}\right) \\
& \in G L_{2}(R) \cap\left(\begin{array}{cc}
1+I & 1+I \\
I & 1+I
\end{array}\right) .
\end{aligned}
$$

We infer that

$$
A=\left(\begin{array}{cc}
s & 0 \\
1-a_{12} t s & a_{12} t
\end{array}\right)^{-1}\left(\begin{array}{cc}
* & 1 \\
* & *
\end{array}\right)\left(\begin{array}{cc}
s a_{12} & 0 \\
1-t s a_{12} & t
\end{array}\right)^{-1} .
$$

Therefore

$$
A^{-1}=\left(\begin{array}{cc}
s a_{12} & 0 \\
1-t s a_{12} & t
\end{array}\right)\left(\begin{array}{cc}
* & 1 \\
* & *
\end{array}\right)^{-1}\left(\begin{array}{cc}
s & 0 \\
1-a_{12} t s & a_{12} t
\end{array}\right) .
$$

From $\left(\begin{array}{ll}* & 1 \\ * & *\end{array}\right) \in G L_{2}(R) \cap\left(\begin{array}{cc}1+I & 1+I \\ I & 1+I\end{array}\right)$, we can find $u \in G L_{1}(I)$ such that

$$
\left(\begin{array}{ll}
1 & 0 \\
* & 1
\end{array}\right)\left(\begin{array}{ll}
* & 1 \\
* & *
\end{array}\right)\left(\begin{array}{ll}
1 & 0 \\
* & 1
\end{array}\right)=\left(\begin{array}{ll}
0 & 1 \\
u & 0
\end{array}\right)=\left(\begin{array}{cc}
0 & u^{-1} \\
1 & 0
\end{array}\right)^{-1}
$$

where $\left(\begin{array}{ll}1 & 0 \\ * & 1\end{array}\right) \in G L_{2}(R) \cap\left(\begin{array}{cc}1 & 0 \\ -1+I & 1\end{array}\right)$. Thus

$$
\left(\begin{array}{ll}
* & 1 \\
* & *
\end{array}\right)^{-1}=\left(\begin{array}{ll}
1 & 0 \\
* & 1
\end{array}\right)\left(\begin{array}{cc}
0 & u^{-1} \\
1 & 0
\end{array}\right)\left(\begin{array}{ll}
1 & 0 \\
* & 1
\end{array}\right)=\left(\begin{array}{cc}
* & u^{-1} \\
* & *
\end{array}\right) .
$$

So we deduce that

$$
\begin{aligned}
A^{-1} & =\left(\begin{array}{cc}
s a_{12} & 0 \\
1-t s a_{12} & t
\end{array}\right)\left(\begin{array}{cc}
* & u^{-1} \\
* & *
\end{array}\right)\left(\begin{array}{cc}
s & 0 \\
1-a_{12} t s & a_{12} t
\end{array}\right) \\
& =\left(\begin{array}{ccc}
* & s a_{12} u^{-1} a_{12} t \\
* & *
\end{array}\right) .
\end{aligned}
$$

As $u \in 1+I$, we have $u^{-1} \in 1+I$. Set $w=s a_{12} u^{-1} a_{12} t$. As $s a_{12} t=1$, we see that $s a_{12} \in 1+I$ is right invertible and $u^{-1} a_{12} t \in 1+I$ is left invertible.

Assume that $B=\left(b_{i j}\right) \in G L_{2}(I)$. Write $B^{-1}=\left(c_{i j}\right)$. Then $B^{-1} \in G L_{2}(I)$; hence, $c_{12} R+c_{11} R=R$ with $c_{12} \in I$. As $I$ is a right quasi-stable ideal, we can find $y \in R$ such that $c_{12}+c_{11} y \in \Gamma(I)$. Obviously, $y \in 1+I$, and so

$$
B^{-1}\left(\begin{array}{ll}
1 & y \\
0 & 1
\end{array}\right)=\left(\begin{array}{cc}
* & c_{12}+c_{11} y \\
* & *
\end{array}\right) \in G L_{2}(R) \cap\left(\begin{array}{cc}
1+I & 1+I \\
I & 1+I
\end{array}\right) .
$$

By the consideration above, we can find some $w_{1} \in 1+I$ such that

$$
\left(\begin{array}{cc}
1 & -y \\
0 & 1
\end{array}\right) B=\left(B^{-1}\left(\begin{array}{ll}
1 & y \\
0 & 1
\end{array}\right)\right)^{-1}=\left(\begin{array}{cc}
* & c_{12}+c_{11} y \\
* & *
\end{array}\right)^{-1}=\left(\begin{array}{cc}
* & w_{1} \\
* & *
\end{array}\right),
$$

where $w_{1}$ is the product of a right invertible element and a left invertible element $v \in 1+I$.

Given $a x+b=1$ with $a, x \in I, b \in R$, then $\left(\begin{array}{cc}1 & x \\ -a & b\end{array}\right)=\left(\begin{array}{cc}1-x a & x \\ -a & 1\end{array}\right)^{-1} \in G L_{1}(I)$. By the proceeding discussion, we can find $z \in R$ such that $\left(\begin{array}{ll}1 & z \\ 0 & 1\end{array}\right)\left(\begin{array}{cc}1 & x \\ -a & b\end{array}\right)=$ 
$\left(\begin{array}{c}* w_{2} \\ * * \\ *\end{array}\right)$, where $w_{2} \in 1+I$ is the product of a right invertible element and a left invertible element $v \in 1+I$. Therefore $x+z b=w_{2}$.

For any regular $x \in I$, it follows from $x y+(1-x y)=1$ that $w:=x+(1-$ $x y) z \in 1+I$ is the product of a right invertible element and a left invertible element $v \in 1+I$. Set $e=x y \in I$. Then $x=x y(x+(1-x y) z)=e w$, where $e=e^{2} \in I$ is an idempotent. Therefore we complete the proof.

Recall that an ideal $I$ of a ring $R$ is regular provided that for any $x \in I$ there exists $y \in I$ such that $x=x y x$. We say that a ring $R$ is right quasi-stable in case it is a right quasi-stable ideal as itself.

Proposition 4.2. Let $I$ be a regular ideal of a ring $R$. If eRe is a right quasistable ring for all idempotents $e \in I$, then $I$ is a right quasi-stable exchange ideal of $R$.

Proof. By [1, Example], $I$ is an exchange ideal. Given $a x+b=1$ with $a \in$ $I, x, b \in R$, then $a=a a^{\prime} a$ for a $a^{\prime} \in R$. Set $c=a^{\prime} a x$. Then $a c+b=1$ with $a, c \in I, b \in 1+I$. As $a, c, 1-b \in I$. In view of [7, Lemma 3.2], there exists an idempotent $e \in I$ such that $a, x, 1-b \in e R e$. Hence, $(1-b)(1-e)=0$, and so $b(1-e)=1-e$. In addition, $(1-b) e=1-b$; hence, $b=b e+1-e$. Thus, $a x+b e=e$. This implies that $b e \in e R e$, and so $e b e=b e$. Since $a x+e b e=e$, by hypothesis, we can find some $u, v, s, t \in e R e$ such that $a+e b e y e=u v, s u=$ $e, v t=e$ for a $y \in R$. Thus, $a+$ beye $+1-e=(u+1-e)(v+1-e)$, and so $a+b($ eye $+1-e)=(u+1-e)(v+1-e)$, where $(s+1-e)(u+1-e)=$ $1,(v+1-e)(t+1-e)=1$ and $s+1-e, t+1-e \in 1+I$. Therefore $I$ is a right quasi-stable ideal of $R$, as desired.

Corollary 4.3. Let $I$ be a regular ideal of a ring $R$. If $a R+b R=R$ with $a \in 1+I, b \in R$ implies that there exists $y \in R$ such that $a+b y \in R$ is right or left invertible, then $I$ is a quasi-stable exchange ideal of $R$.

Proof. Let $e \in I$ be an idempotent. In view of [5, Lemma 4.1], $e$ Re is one-sided unit-regular. For any $x \in e R e$, by [3, Theorem 4], there exist an idempotent $f \in e R e$ and a right or left $u \in e R e$ such that $x=e u$. This implies that $e R e$ is a right quasi-stable ring from Theorem 2.2. According to Proposition 4.2, $I$ is a right quasi-stable exchange ideal. By the symmetry of one-sided unitregularity, we establish the result.

Recall that an ideal $I$ of a regular ring $R$ satisfies the comparability axiom provided that for any $x, y \in I$, either $x R \lesssim y R$ or $y R \lesssim x R$ (cf. [10]). Let $I$ be an ideal of a regular ring $R$. If $I$ satisfies the comparability axiom, we note that $a R+b R=R$ with $a \in 1+I, b \in R$ implies that $a+b y \in R$ is right or left invertible for a $y \in R$.

Corollary 4.4. Let $I$ be a regular ideal of a ring $R$. If I satisfies the comparability axiom, then $I$ is quasi-stable. 
Proof. Clearly, $a R+b R=R$ with $a \in 1+I, b \in R$ implies that $a+b y \in R$ is right or left invertible. Therefore we complete the proof by Corollary 4.3.

By [8, Corollary 9.15], every regular, right self-injective ring satisfies general comparability. We now extend this result to right injective ideals of regular rings.

Proposition 4.5. Let I be a regular ideal of a ring $R$. If $I$ is an injective right $R$-module, then $I$ is a quasi-stable ideal of $R$.

Proof. Since $I$ is regular, $I$ is an exchange ideal. As $I$ is injective, there exists a splitting exact sequence $0 \rightarrow I \hookrightarrow R \rightarrow R / I \rightarrow 0$. Thus, we have a right $R$-module $C \cong R / I$ such that $R=I \oplus C$. Thus, $I=e R$ for some idempotent $e \in I$. Let $f \in I$ be an idempotent. Then we have an inclusion $i: f R \hookrightarrow e R$. Construct a $R$-morphism $\varphi: e R \rightarrow f R$ given by $\varphi(e r)=f e r$ for any $r \in R$. It is easy to verify that $\varphi i=1_{f R}$. This implies that the exact sequence $0 \rightarrow$ $f R \hookrightarrow e R \rightarrow e R / f R \rightarrow 0$ splits. Thus, we have a right $R$-module $D \cong e R / f R$ such that $e R=f R \oplus D$. Since $e R$ is injective, so is $f R$. For any $m \in Z(f R)$, there exists some $z \in R$ such that $m=m z m$. Hence, $r(m)=(1-z m) R$. As $r(m) \bigcap z m R=0$, we get $z m R=0$; hence, $m=m z m=0$. That is, $Z(f R)=0$, i.e., $f R$ is nonsingular. In view of [8, Corollary 1.23], $f R f \cong \operatorname{End}_{R}(f R)$ is a regular, right self-injective ring. According to [8, Corollary 9.15], eRe satisfies general comparability. Let $x \in f R f$, we can find an idempotent $g \in f R f$ and a related unit $w \in f R f$ such that $x=g w$. As $w \in f R f$ is a related unit, there exists an idempotent $g \in f R f$ such that $g w \in g(f R f)$ is right invertible and $(f-g) w \in(f-g)(f R f)$ is left invertible. Thus, $w=((f-g) w+g)(g w+f-g)$. According to Theorem 2.2, $e R e$ is a right quasi-stable ring. According to Proposition 4.2, $I$ is a right quasi-stable ideal. Analogously, we show that $I$ is a left quasi-stable ideal. Therefore $I$ is quasi-stable, as desired.

Let $R$ be a regular ring, and let $a \in R$. If $R a R$ is injective, it follows from Proposition 4.5 and Theorem 2.2 that $a$ is the product of an idempotent, a left invertible element and a right invertible element.

Example 4.6. Let $R$ be regular, and let

$$
I=\{x \in R \mid x R \text { is injective }\} .
$$

Then $I$ is a quasi-stable ideal.

Proof. It is directly proved that $I$ is an ideal of $R$. For any $a \in I$, there exists an idempotent $e \in I$ such that $a \in e R e$ from [6, Lemma 3.2]. As $e R$ is injective, it follows from [8, corollary 1.23] that $e R e$ is a regular, right selfinjective ring. Thus, it satisfies related comparability. Hence, there exists an idempotent $f \in e R e$ and a related unit $w \in$ ere such that $a=e u$. This implies that $a=e(u+1-e)$, where $e \in I$ is an idempotent and $u+1-e \in \Gamma(I)$. According to Theorem 2.2, $I$ is a right quasi-stable ideal. Similarly, we show that $I$ is a left quasi-injective ideal, as asserted. 


\section{Directly finite ideals}

We say that an ideal $I$ of a $\operatorname{ring} R$ is directly finite provided that for any $a, b \in I,(1+a)(1+b)=1$ implies that $(1+b)(1+a)=1$. An ideal $I$ of a ring $R$ is said to be of bounded index provided that there exists some $n \in \mathbb{N}$ such that $x^{n}=0$ for any nilpotent element $x \in I$. Let $R$ be a regular ring, and let $I=\left\{x \in R \mid \operatorname{End}_{R}(x R)\right.$ is of bounded index $\}$. Then $I$ is a directly finite, quasi-stable exchange ideal.

Lemma 5.1. Let $I$ be a directly finite, right quasi-stable exchange ideal of a ring $R$. Suppose that $A X+B=I_{n}$ with $A, X \in M_{n}(I), B \in M_{n}(R)$. Then

(1) There exists some $Y \in M_{n}(R)$ such that $A+B Y \in G L_{n}(I)$.

(2) There exists some $Z \in M_{n}(R)$ such that $X+Z B \in G L_{n}(I)$.

Proof. (1) Since $I$ is directly finite, one easily checks that $\Gamma(I)=G L_{1}(I)$. By iteration of the process of Theorem 3.2 and replacing the elements in $\Gamma(I)$ by invertible elements in $1+I$, we can find some $Y \in M_{n}(R)$ such that $A+B Y \in$ $G L_{n}(I)$.

(2) By (1), there is $Y \in M_{n}(R)$ such that $A+B Y \in G L_{n}(I)$. In view of Lemma 2.4, one directly verifies that $\left(X+\left(X_{n}-X Y\right)(A+B Y)^{-1} B\right)^{-1}=$ $A+Y\left(I_{n}-X A\right)$. Check $Z=\left(X_{n}-X Y\right)(A+B Y)^{-1}$. Then $X+Z B \in G L_{n}(I)$, as asserted.

Theorem 5.2. Let $I$ be a directly finite, right quasi-stable exchange ideal of a ring $R$. Then for any regular $A \in M_{n}(I)$ there exist $U, V \in G L_{n}(I)$ such that $U A V=\operatorname{diag}\left(e_{1}, \ldots, e_{n}\right)$ for some idempotents $e_{1}, \ldots, e_{n} \in I$.

Proof. Given any regular matrix $A \in M_{n}(I)$, there exists $E=E^{2} \in M_{n}(I)$ such that $A M_{n}(R)=E M_{n}(R)$. Similarly to Theorem 3.4, we have idempotents $e_{1}, \ldots, e_{n} \in I$ such that $\varphi: A M_{n}(R) \cong \operatorname{diag}\left(e_{1}, \ldots, e_{n}\right) M_{n}(R)$. Then $M_{n}(R) A=M_{n}(R) \varphi(A), \varphi(A) M_{n}(R)=\operatorname{diag}\left(e_{1}, \ldots, e_{n}\right) M_{n}(R)$. One directly verifies that there exist some $X, Y \in M_{n}(I)$ such that $X A=\varphi(A)$ and $A=Y \varphi(A)$. Since $Y X+\left(I_{n}-Y X\right)=I_{n}$, it follows by Lemma 5.1 that there exists some $Z \in M_{n}(R)$ such that $U:=X+Z\left(I_{n}-Y X\right) \in G L_{n}(I)$. Hence $U A=\left(X+Z\left(I_{n}-Y X\right)\right) A=X A=\varphi(A)$. Likewise, we can find some $V \in G L_{n}(I)$ such that $\varphi(A) V=\operatorname{diag}\left(e_{1}, \ldots, e_{n}\right)$. Therefore $U A V=$ $\operatorname{diag}\left(e_{1}, \ldots, e_{n}\right)$, as asserted.

Let $I$ be an ideal of a ring $R$. Set $B(I)=\left\{e \in I \mid e=e^{2}\right.$ and $e x=$ $x e$ for any $x \in I\}$. We say that $I$ is an abelian ideal in case every idempotent in $I$ is in $B(I)$. For example, every semicommutative ideal of a ring is an abelian ideal.

Corollary 5.3. Let $I$ be an abelian exchange ideal of a ring $R$. Then for any regular $A \in M_{n}(I)$ there exist $U, V \in G L_{n}(I)$ such that $U A V=\operatorname{diag}\left(e_{1}, \ldots, e_{n}\right)$ for some idempotents $e_{1}, \ldots, e_{n} \in I$. 
Proof. For any regular $x \in I$, we have $y \in I$ such that $x=x y x$ and $y=y x y$. Since $I$ is an abelian exchange ideal of $R$, we have $x=x^{2} y=y x^{2}$, and then $x=x y(1+x-x y)$. Set $e=x y$ and $u=1+x-x y$. Then $e=e^{2} \in I$ and $u(1+y-x y)=1$. Hence $u \in \Gamma(I)$. Thus $I$ is a right quasi-stable ideal by Theorem 2.2.

Suppose that $(1-x)(1-y)=1$ with $x, y \in I$; hence, $(1-y)(1-x) \in 1+I$ is an idempotent. Since $I$ is an abelian ideal of $R,(1-(1-y)(1-x)) x=x(1-(1-$ $y)(1-x))$. Furthermore, we get $(1-y)(1-x)(1-x)=(1-x)(1-y)(1-x)$. So $(1-y)(1-x)=(1-y)(1-x)(1-x)(1-y)=(1-x)(1-y)(1-x)(1-y)=1$. That is, $I$ is a directly finite ideal. Therefore we complete the proof from Theorem 5.2.

Recall that an ideal $I$ of a ring $R$ is periodic provided that for any $x \in I$, there exists $n(x) \in \mathbb{N}$ such that $x=x^{n(x)+1}$.

Corollary 5.4. Let $I$ be a periodic ideal of a ring $R$. Then for any regular $A \in M_{n}(I)$ there exist $U, V \in G L_{n}(I)$ such that $U A V=\operatorname{diag}\left(e_{1}, \ldots, e_{n}\right)$ for some idempotents $e_{1}, \ldots, e_{n} \in I$.

Proof. For any idempotent $e \in I$ and any idempotent $x \in I$, we have $(e x-$ $e x e)^{2}=0$. So we deduce that $e x=e x e$ because $I$ is an periodic ideal. Likewise, we have $x e=e x e$; hence, $e x=x e$. This means that $I$ is an abelian ideal of $R$. On the other hand, $I$ is a strongly $\pi$-regular ideal; hence, it is an exchange ideal. So the proof is true by Corollary 5.3.

Example 5.5. Let $V$ be a countably generated infinite-dimensional vector space over a division $D$, and let $\left(a_{i j}\right) \in M_{n}\left(\operatorname{End}_{D}(V)\right)$ with all $\operatorname{dim}_{D}\left(a_{i j} V\right)<$ $\infty$. Then there exist $U, V \in G L_{n}\left(\operatorname{End}_{D}(V)\right)$ such that $U A V=\operatorname{diag}\left(e_{1}, \ldots, e_{n}\right)$ for some idempotents $e_{1}, \ldots, e_{n} \in \operatorname{End}_{D}(V)$.

Proof. Let $I=\left\{x \in \operatorname{End}_{D}(V) \mid \operatorname{dim}_{D}(x V)<\infty\right\}$. Obviously, $I$ is an ideal of $\operatorname{End}_{D}(V)$. For any idempotent $e \in I$, eRe is unit-regular; hence, $I$ has stable range one. This implies that $I$ is directly finite. In view of Proposition 2.5, $I$ is a quasi-stable ideal. According to Theorem 5.2, the result follows.

Let $R$ be an exchange ring, and let $\left(a_{i j}\right) \in M_{n}(R)$. If each $R a_{i j} R$ has stable range one, analogously, we conclude that there exist $\left(u_{i j}\right),\left(v_{i j}\right) \in G L_{n}(R)$ such that $\left(u_{i j}\right)\left(a_{i j}\right)\left(v_{i j}\right)=\operatorname{diag}\left(e_{1}, \ldots, e_{n}\right)$ for some idempotents $e_{1}, \ldots, e_{n} \in R$. In addition, $R u_{i j} R, R v_{i j} R(i \neq j), R\left(1-u_{i i}\right) R$ and $R\left(1-v_{i i}\right) R$ all have stable range one.

\section{References}

[1] P. Ara, Extensions of exchange rings, J. Algebra 197 (1997), no. 2, 409-423.

[2] P. Ara, K. R. Goodearl, K. C. O'Meara, and E. Pardo, Diagonalization of matrices over regular rings, Linear Algebra Appl. 265 (1997), 147-163.

[3] H. Chen, Elements in one-sided unit regular rings, Comm. Algebra 25 (1997), no. 8, $2517-2529$. 
[4] _ Generalized stable regular rings, Comm. Algebra 31 (2003), no. 10, 4899-4910.

[5] _, $2 \times 2$ invertible matrices over weakly stable rings, J. Korean Math. Soc. 46 (2009), no. 2, 257-269.

[6] H. Chen and M. Chen, Generalized ideal-stable regular rings, Comm. Algebra 31 (2003), no. $10,4989-5001$.

[7] _ On products of three triangular matrices over associative rings, Linear Algebra Appl. 387 (2004), 297-311.

[8] K. R. Goodearl, Von Neumann Regular Rings, Pitman, London, San Francisco, Melbourne, 1979; second ed., Krieger, Malabar, Fl., 1991.

[9] T. Y. Lam, A crash course on stable range, cancellation, substitution and exchange, J. Algebra Appl. 3 (2004), no. 3, 301-343.

[10] D. Lu, Q. Li, and W. Tong, Comparability, stability, and completions of ideals, Comm. Algebra 32 (2004), no. 7, 2617-2634.

[11] K. C. O'Meara and R. Raphael, Uniform diagonalisation of matrices over regular rings, Algebra Universalis 45 (2000), 383-405.

[12] F. Perera, Lifting units modulo exchange ideals and $C^{*}$-algebras with real rank zero, J. Reine Angew. Math. 522 (2000), 51-62.

Department of Mathematics

HANGZHou Normal University

Hangzhou 310036, P. R. China

E-mail address: huanyinchen@yahoo.cn 